\title{
Assessment of the Dermal and Ocular Irritation Potential of Lomefloxacin by Using In Vitro Methods
}

\author{
Jun-Ho Ahn, Ki-Hwan Eum and Michael Lee \\ Department of Biology, College of Natural Sciences, University of Incheon, Incheon 406-772, Korea
}

(Received December 2, 2009; Revised December 18, 2009; Accepted December 30, 2009)

\begin{abstract}
The evaluation of eye and skin irritation potential is essential to ensuring the safety of human in contact with a wide variety of substances. Despite this importance of irritation test, little is known with respect to the irritation potency of lomefloxacin, a fluoroquinolone antibiotic, which has been known to cause phototoxicity with an abnormal reaction of the skin. Thus, to investigate the tendency of lomefloxacin to cause eye and skin irritation, we carried out in vitro eye irritation test using Balb/c 3T3, and in vitro skin irritation test using KeraSkin ${ }^{\mathrm{TM}}$ human skin model system. 3T3 neutral red uptake assay has been proposed as a potential replacement alternative for the Draize Eye irritation test. In this study, the $\mathrm{IC}_{50}$ value obtained for lomefloxacin was $375 \mu \mathrm{g}$. According to the classification model used for determining in vitro categories, lomefloxacin was classified as moderately irritant. For evaluation of skin irritation, engineered epidermal equivalents (KeraSkin ${ }^{\mathrm{TM}}$ ) were subjected to 10 and $25 \mathrm{mg}$ of lomefloxacin for 15 minutes. Tissue damage was assessed by tissue viability evaluation, and by the release of a pro-inflammatory mediator, interleukin$1 \alpha$. Lomefloxacin increased the interleukin- $1 \alpha$ release after 15 minutes of exposure and 42 hours of post incubation, although no decrease in viability was observed. Therefore, lomefloxacin is considered to be moderately irritant to skin and eye.
\end{abstract}

Key words: Lomefloxacin, Eye irritation, Skin irritation, In vitro testing, Human skin model

\section{INTRODUCTION}

The one of the physiological response of the exposition and accidental contact with new chemical entities (NEC) is irritation to eye and skin, which involves local redness and oedema. Thus, assessment of eye and skin irritation potential is an important part of preclinical safety assessment for NEC before humans can be exposed to such substances. Until now, the evaluation of eye and skin irritation potential is largely based on animal experiments. Especially, the Draize rabbit eye and skin irritancy tests have been the standard for prediction of the human ocular and dermal irritation for decades since Draize's techniques were used by the Food and Drug Administration to evaluate the safety of several substances (Fitzhugh et al., 1946). However, the inadequacies of the Draize test and the ethical concerns involving the use of laboratory animals have led to the development of in vitro alternative methods to replace it.

Until now a large number of studies have been under-

Correspondence to: Michael Lee, Department of Biology, College of Natural Sciences, University of Incheon, 12-1 Songdo-dong, Yeonsu-gu, Incheon 406-840, Korea

E-mail: mikelee@incheon.ac.kr taken to find tests that replace the need for animals in eye safety testing (Vinardell and Mitjans, 2008). The following different methods have been proposed as alternatives: Red Blood Cell Test (Pape et al., 1987), the Haemoglobin Denaturation Test (Hatao et al., 1999), the Hen's Egg TestChorioallantoic Membrane assay (Luepke, 1985), Isolated Cornea Opacity and Permeability Test (Gautheron et al., 1992), The Enucleated Eye Test (Prinsen and Koeter, 1993), and MTT mitochondrial reduction with cell culture (Yang and Acosta, 1994). Among these tests, the 3T3 neutral red uptake (NRU) assay has been found to be efficient and costeffective replacement alternative. The 3T3 NRU assay was included as part of the COLIPA (European Cosmetic, Toiletry and Perfumery Association) Validation Project on Alternatives to the Draize eye irritation (Brantom et al., 1997). In this test, a decrease in cell number, as measured by uptake of the dye neutral Red, serves as an indicator of potential cytotoxicity.

Additionally, in order to evaluate the skin irritancy of test articles, a useful approach using in vitro testing methods with human skin model system is increasingly used instead of Driaze skin irritation test. Various in vitro models of the epidermis (epidermal equivalents) are commercially available today. In general, these engineered epidermal equiva- 
lents consist of normal, human-derived keratinocytes, which have been cultured on porous, flexible membranes of cell culture inserts at the air-liquid interface to form a multilayered, highly differentiated model of the human epidermis (Rosdy and Clauss, 1990). In this study, KeraSkin ${ }^{\mathrm{TM}}$ from Modern Cell \& Tissue Technologies (MCTT) Inc. (Seoul, Korea) was used as an in vitro model of the epidermis. KeraSkin skin irritation test consists in putting a sufficient amount of sample preparation on the surface of the epidermis model. After a certain incubation period, cell viability is assessed with the use of MTT ((3-4,5 dimethyl triazole 2yl) 2,5-diphenyltetrazoliumbromide) colorimetric test. Additionally, the release of a proinflammatory mediator, interleukin- $1 \alpha$ (IL- $1 \alpha)$, is measured as a marker of the onset of tissue damage.

Lomefloxacin, one of a fluoroquinolone antibiotic, is used to treat bacterial infections including bronchitis and urinary tract infections, but is recognized to be associated with drug-induced phototoxicity including an abnormal reaction of the skin to light sources (Ball et al., 1999; Chetlat et al., 1996; Hong et al., 2005; Kim et al., 2006). Phototoxicityinduced DNA damage may lead to mutated skin cells, which in turn can contribute to an elevated skin cancer risk. Despite of these known phototoxicity, the potential of skin and eye irritation for lomefloxacin has not been well studies.

The aim of present study was therefore to investigate the tendency of lomefloxacin to cause eye and skin irritation. Thus, we carried out in vitro eye irritation test using Balb/c $3 \mathrm{~T} 3$, and in vitro skin irritation using KeraSkin ${ }^{\mathrm{TM}}$ human skin model system. Overall the data indicate that lomefloxacin is likely to be moderately irritant to skin and eye.

\section{MATERIALS AND METHODS}

Test article, chemicals and engineered epidermal equivalent. Lomefloxacin (CAS\# 98079-51-7) were obtained from Sigma-Aldrich (St. Louis, MO). Lomefloxacin was dissolved in distilled water and serially diluted to the appropriate concentrations immediately before use. Most chemicals including MTT, Neutral Red dye, and sodium dodecyl sulfate (SDS) were obtained from Sigma (St. Louis, MO). MEM medium, RPMI1640 medium, fetal bovine serum, and penicillin-streptomycin were purchased from GIBCOInvitrogen (Carlsbad, CA). A commercially available human epidermal equivalent, KeraSkin ${ }^{\mathrm{TM}}$ (MM311, MCTT Inc., Seoul, Korea), was used as an in vitro model of the epidermis in this study. This model (diameter $=8 \mathrm{~mm}$ ) consists of normal human-derived keratinocytes, multiple viable cell layers, functional stratum corneum.

Cell lines and cell culture. Balb/c 3T3, clone 31 (mouse fibroblast cell line) (Aaronson and Todaro, 1968) for in vitro eye irritation test were obtained from American Type Culture Collection (Manassas, VA). The cells were cultured in Dulbecco's Modified Eagle's Medium supplemented with $100 \mathrm{U}$ penicillin, $100 \mu \mathrm{g} / \mathrm{m} l$ streptomycin, $2 \mathrm{mM}$ L-glutamine, and $10 \%$ calf serum. Sub-culture was conducted every $2 \sim 3$ days so as to prevent overgrowth.

In vitro eye irritation test - neutral red uptake cytotoxicity assay. In vitro eye irritation test was performed using Balb/c 3T3, clone 31 as described by Test Method Protocol from NICEATM (The National Toxicology Program (NTP) Interagency Center for the Evaluation of Alternative Toxicological Methods) (NICEATM, 2003) and Lee et al. (Lee et al., 2008) with minor modifications. Briefly, the cells were plated into 96-well microliter plates (Costar, Cambridge, MA) at $1 \times 10^{4}$ cells/well and then incubated for $24 \mathrm{~h}$ at $37^{\circ} \mathrm{C}$ in a humidified $5 \% \mathrm{CO} 2 / 95 \%$ air incubator. Cells were then exposed to dilutions (six replicate wells per concentration) of lomefloxacin for $24 \mathrm{~h}$. Meanwhile, a positive control group was set up with SDS. After washing with $200 \mu l$ of PBS three times, the cells were incubated with $100 \mu l$ of Neutral Red (NR) $(50 \mu \mathrm{g} / \mathrm{m} l)$ per well for $3 \mathrm{~h}$. NR medium were removed and the cells were washed with $150 \mu l$ of Earle's balanced salt solution. $150 \mu l$ of NR desorb solution (water : ethanol : acetic acid $=49: 50: 1$ ) was added to cells with shaking for $10 \mathrm{~min}$. The optical density of the NR extract was measured at $540 \mathrm{~nm}$ using a microtiter plate reader (Molecular Devices, Sunnyvale, CA). The concentration producing $50 \%$ inhibition for neutral red uptake was calculated. The concentrations of $\geq 1250 \mu \mathrm{g} / \mathrm{ml}$, $200 \mu \mathrm{g} / \mathrm{m} l \leq \mathrm{IC}_{50}<1250 \mu \mathrm{g} / \mathrm{m} l$ and $<200 \mu \mathrm{g} / \mathrm{m} l$ represented non-/mildly irritant, moderately irritant and strongly irritant respectively (Yang et al., 2007).

In vitro skin irritation test. Upon receipt, the KeraSkin samples were transferred to 6 -well plates containing $900 \mu l$ per well of culture medium (MCTT Inc.), according to recommendations from the supplier. After overnight culture at $37^{\circ} \mathrm{C}$ and $5 \% \mathrm{CO}_{2}$ the samples were transferred to fresh medium and directly used in an experiment. In vitro skin irritation test was performed according to the method of OECD draft proposal (OECD, 2007) and Jung et al. (Jung et al., 2008) with minor modifications. Epidermis equivalents were directly exposed in triplicate to $25 \mathrm{mg}$ of topically applied lomefloxacin for $15 \mathrm{~min}$ (Osborne et al., 2000). Following exposure, lomefloxacin were removed by repeated rinsing with phosphate buffered saline and the equivalents placed in fresh medium. After $42 \mathrm{hr}$ post-treatment the medium underneath the KeraSkin samples was collected from each well and stored at $-80^{\circ} \mathrm{C}$ for extracellular IL- $1 \alpha$ analysis. The samples were washed twice with PBS and used in a viability assay.

Viability measurement: The viability of the KeraSkin samples ( $n=3$ per treatment group) was determined by a colorimetric MTT assay (MTT-100, MatTek Corporation, Ashland, MA, USA). A volume of $300 \mu l$ of MTT solution 
was added to all test wells containing KeraSkin samples, and the plates were incubated at $37^{\circ} \mathrm{C}$ and $5 \% \mathrm{CO}_{2}$ for a period of $3 \mathrm{~h}$. After incubation, the samples were removed from the MTT plates and submerged them in $2 \mathrm{ml}$ of isopropyl alcohol per well. For all samples, the extractant solution was pipetted up and down to ensure complete mixing, and finally, $200 \mu l$ were transferred to a 96-well plate for measuring the optical density (OD) using a plate reader set to $570 \mathrm{~nm}$. Values of the blank extractant were subtracted from all sample values.

IL- $\alpha$ measurement: The levels of IL- $1 \alpha$ were determined in the medium of all KeraSkin samples by Quantikine Human IL-1 $\alpha / \mathrm{IL}-1 \mathrm{~F} 1$ Immunoassay (R\&D systems, Minneapolis, MN). Briefly, $200 \mu l$ medium or test standard were added into the appropriate pre-coated wells and incubated for $2 \mathrm{~h}$ at room temperature. After washing each well, $200 \mu l$ of IL- $1 \alpha$ conjugate was added, and the plates were incubated at room temperature for an additional $1 \mathrm{~h}$. Following the washing step, $200 \mu l$ of a substrate solution containing tetramethylbenzidine and hydrogen peroxide was added to each well. After incubation for $20 \mathrm{~min}$ at room temperature, $50 \mu l$ of stop solution containing $2 \mathrm{~N}$ sulfuric acid was added to each well. The optical density of each well was measured at $450 \mathrm{~nm}$ using a microtiter plate reader. To correct for optical imperfections in the well plate, wavelength correction was performed at $570 \mathrm{~nm}$. The concentration of IL- $1 \alpha$ in the medium samples was calculated using the obtained standard curve. Duplicate measurements were performed for each individual sample.

Interpretation of results: According to the draft OECD test guideline (OECD, 2007), the test article was considered to be irritant to skin if the viability after 15 minutes of exposure and 42 hours of post incubation is more $(>)$ than $50 \%$, and the amount of IL- $1 \alpha$ release is more $(>)$ than $60 \mathrm{pg} / \mathrm{ml}$. The test substance is considered to be non irritant to skin if the viability after 15 minutes of exposure and 42 hours of post incubation is more $(>)$ than $50 \%$, and the amount of IL- $1 \alpha$ release is less or equal $(\leq)$ to $60 \mathrm{pg} / \mathrm{ml}$.

Statistical analysis. Data are presented as means and standard deviations and the means of percentage of viability was compared by one-way analysis of variance and Dunnett Multiple Comparisons were performed for comparison of the vehicle control and test item-treated groups. $P<0.05$ was considered to be the level of significance. Statistical analysis was performed using GraphPad InStat 3.

\section{RESULTS}

\section{In vitro eye irritation test - neutral red uptake assay.}

3T3-NRU assay has been proposed as a potential replacement alternative for the Draize Eye irritation test. This test is a short-term test system based on a monolayer culture of $3 \mathrm{~T} 3$ cells with cytotoxicity used as the endpoint for eye irri- tancy evaluation of test article. According to Test Method Protocol from NICEATM (NICEATM, 2003), it is recommended that the maximum soluble dose should be selected as the highest test concentration. On the other hand, in case of in vitro methods, it is advisable to test at more than one concentration with visible precipitation. Thus, a concentration level of $20 \mathrm{mg} / \mathrm{m} l$ was selected as the highest test concentration due to precipitation of the lomefloxacin in culture medium (solubility limit: $5 \mathrm{mg} / \mathrm{m} l$ in culture medium). The main study was performed where the final concentrations of lomefloxacin ranged between 1 and $20 \mathrm{mg} / \mathrm{m} l$ with 2 fold intervals between test points. SDS, as a positive control, was assayed at 4 concentrations with a 2 fold dilution factor using $0.1 \%$ as a maximum concentration. $\mathrm{The} \mathrm{IC}_{50}$ value obtained for lomefloxacin was $375 \mu \mathrm{g}$. According to the classification model used for the determination of in vitro categories, lomefloxacin was classified as moderately irritant. As expected, there was a significant inhibition in MTT viability in the positive control, SDS treatment.

In vitro skin irritation test. In vitro skin irritation test was performed with the KeraSkin human model system. The parameters determined in vitro were the percent MTT

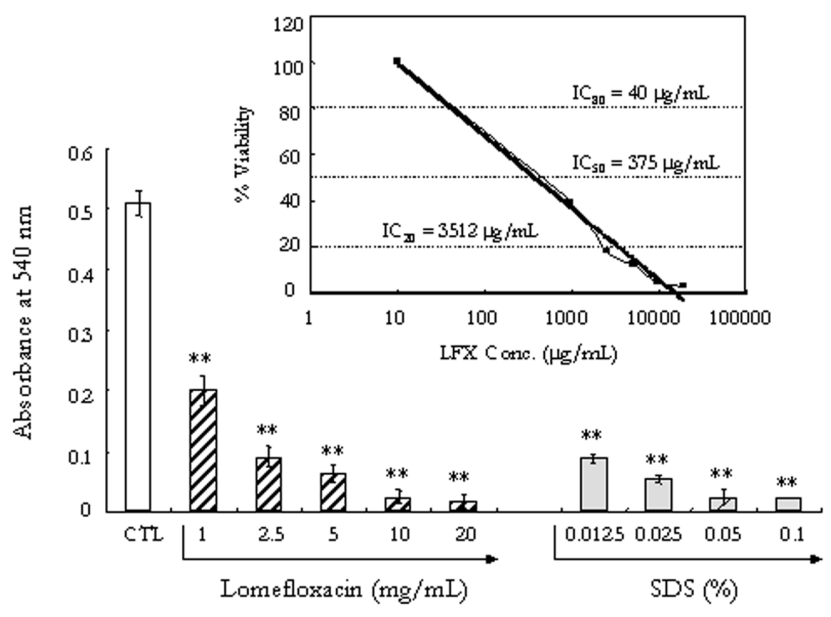

Fig. 1. In vitro eye irritatioin test - Neutral Red Uptake assay. In vitro eye irritation test was performed using Balb/c 3T3 cell line. The cells were treated with increasing concentrations of lomefloxacin ranged from 1 to $20 \mathrm{mg} / \mathrm{m} /$ and then incubated in 96 well plates for $24 \mathrm{~h}$ before Neutral Red. A positive control group was set up with sodium dodecyl sulphate (SDS) ranged from 0.0125 to $0.1 \%$. After an additional $3 \mathrm{~h}$ incubation periods the optical density of the NR extract was measured at $540 \mathrm{~nm}$ using a microtiter plate reader. The concentration producing $50 \%$ inhibition for neutral red uptake was calculated. Values represent the mean \pm SD of duplicate determinants from one of three representative experiments. ${ }^{*} P<0.01$ as determined by Dunnett Multiple Comparisons test as compared to vehicle control group. Inset, A plot of the log of the \% viability versus concentration of lomefloxacin in order to the $\mathrm{IC}_{50}$ value. 


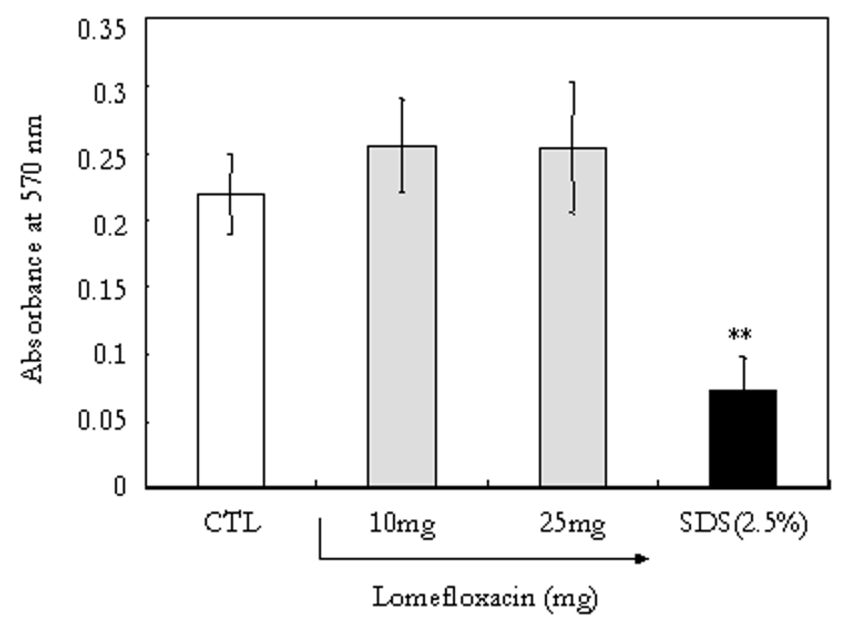

Fig. 2. In vitro skin irritation test - Cell viability in response to lomefloxacin application. KeraSkin equivalents were treated with vehicle or with $10 \mathrm{mg}$ or $25 \mathrm{mg}$ preparations of lomefloxacin as described in Materials and Methods. Each column represents the mean viability (as \% of vehicle controls) and bars indicate the standard deviation $(\mathrm{n}=3)$. ${ }^{* *} P<0.01$ as determined by Dunnett multiple test as compared to vehicle control group.

cell viability at the end of each exposure time as well as the amount of the cytokine IL- $1 \alpha$ released into the culture media collected at the end of each exposure period. As described by US patent 6020148 (Osborne et al., 2000), $25 \mathrm{mg}$ was selected as the highest concentration for dry powder.

MTT assay: The tissue viability, an indication of the amount of tissue damage, was quantitatively assessed by a MTT assay. The results of the in vitro MTT viability test of lomefloxacin are shown in Fig. 2. The viability results imply that all KeraSkin samples were resistant to lomefloxacin application. No decreases in viability were found in both treatment groups (10 mg: $98.8 \%$ viable, $25 \mathrm{mg}$ : $107 \%$ viable) after 15 minutes of exposure and 42 hours of post incubation. Thus, lomefloxain was concluded to be non-irritant in this assay at up to the highest feasible concentration which could be evaluated. As expected, there was a significant inhibition in MTT viability in the positive control, $2.5 \%$ SDS treatment ( $25.5 \%$ viable).

IL-1 $\alpha$ measurement: For epidermis tissues showing a cell viability $>50 \%$, the amount of IL- $1 \alpha$ released into the tissue culture medium at the end of the post-treatment incubation period (after $42 \mathrm{~h}$ post-treatment incubation) was assessed by a quantitative enzyme immunoassay technique, in order to detect the damaging effects of lomefloxacin. The absolute values of IL- $1 \alpha$ release, expressed in $\mathrm{pg} / \mathrm{m} l$, are shown in Fig. 3. In contrast with the obtained MTT viability results, an increase in IL- $1 \alpha$ release was observed in 10and $25-\mathrm{mg}$ treatment groups after 15 minutes of exposure and 42 hours of post incubation. This effect was most pronounced in the $25 \mathrm{mg}$ treated groups in which an 8.76-fold

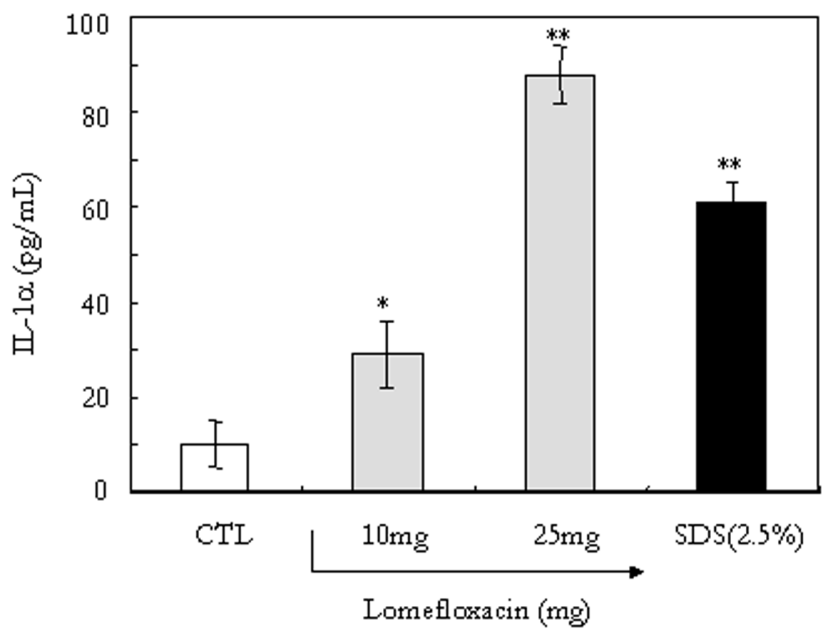

Fig. 3. In vitro skin irritation test - Interleukin-1 $\alpha$ secretion from epidermal equivalents in response lomefloxacin application. KeraSkin equivalents were treated with vehicle or lomefloxacin. The levels of IL-1 $\alpha$ in the medium of all KeraSkin samples were analysed by Quantikine Human IL-1 $\alpha /$ IL-1F1 Immunoassay. Each column represents the mean of cytokine secretion and bars indicate the standard deviation $(n=3)$. Results of one representative experiment of three are shown. ${ }^{* *} P<0.01$ and ${ }^{*} P<0.05$ as determined by Dunnett multiple test as compared to vehicle control group.

increase was observed in comparison with the vehicle control groups $(p<0.05)$.

\section{DISCUSSION}

The primary objective of this study was to evaluate the irritant potency of lomefloxacin, which has been used to treat bacterial infections. The previous results have shown that lomefloxacin could enhance UV-induced DNA damage and skin carcinogenesis (Ball et al., 1999; Chetlat et al., 1996). However, a direct effect on eye and skin remains to be fully elucidated. In general, the Draize rabbit eye and skin irritancy tests have been used to determine the human ocular and dermal irritation for NEC during a pre-clinical safety evaluation. Despite these widespread use in irritation test, the high number of variables in the Draize test limits its reproducibility (York and Steiling, 1998), especially for moderately irritating compounds. Especially, animals of the same strain can respond in various ways to a given compound, and different examiners can obtain diverse results from the same test (Freeberg et al., 1986; Griffith et al., 1980). Additionally, Draize eye and skin irritation test is time-consuming and costly with many animals. More importantly, several scientists state that rabbit responses cannot accurately be used to predict human responses due to the difference between the sensitivity in rabbit skin and human skin (Calvin, 1992).

In the past few years some effort has been put into the 
development of several short-term in vitro tests for prediction of eye and skin irritation in response to drug application. Although results indicated that in vitro short-term tests did not necessarily predict effects in the Draize test, the 3T3-NRU assay appeared to be efficient and cost-effective methods for in vitro alternative eye irritation tests. The neutral red uptake assay, which is based on the ability of viable cells to incorporate and bind the supravital dye neutral red in the lysosomes, provides a quantitative estimation of the number of viable cells in a culture. The NRU assay was included as part of the COLIPA international validation trial of in vitro alternatives to the Draize eye irritation test. As based on the results of the 3T3-NRU assay, lomefloxacin has a moderate eye irritation potential. However, some previous study showed that, as a stand-alone assay, the NRU assay is of limited utility as a Draize replacement (Spielmann et al., 1995). Thus, the way forward for screening of eye-irritant substances from in vitro the 3T3-NRU assays is more likely to come from a combination of the results of several different assays including Hen's Egg Test-Chorioallantoic Membrane test.

In the Draize skin irritation test, the product is applied to the shaved skin of rabbit, and the appearance of oedema and/or erythema is evaluated at $1,24,48$, and $72 \mathrm{~h}$ after application. Recently, reconstructed human Epidermis (RhE) test method was developed for evaluating skin irritation. The RhE test method has been shown to be relevant and reliable with a sensitivity of $90 \%$ and a specificity of $80 \%$ (Alépée et al., 2009). In this method, the main selected endpoint is the cell viability (MTT reduction), with a threshold of $50 \%$ viability. Additionally, IL- $1 \alpha$ release into the assay medium is evaluated as a promising complementary endpoint to the classic MTT cytotoxicity test (Mosman, 1983). Previous research demonstrated that IL- $1 \alpha$ is an important inflammatory mediator in the skin (Coquette et al., 2003). Currently, IL- $1 \alpha$ is widely accepted for screening the damaging potential of chemical irritants before the physiological signs of skin irritation occur (Corsini et al., 1996). Conversely, the recent study reported that the RhE test method was not improved by integrating another endpoint such as IL-1 $\alpha$ (Alépée et al., 2009). In our study, no decreases in viability were found in lomefloxaxcin treatment groups while an increase in IL- $1 \alpha$ release was observed after 15 minutes of exposure and 42 hours of post incubation of lomefloxacin.

Taken together, lomefloxain was concluded to be moderately irritant in our in vitro studies. However, no alternative in vitro tests for both ocular and skin irritation are available for regulatory purposes. Thus, it will be important to establish battery of tests, as no single assay can fulfill the requirements for risk assessment of eye and skin safety using an in vitro method.

\section{ACKNOWLEDGMENT}

This work was supported by a University of Incheon
Research Grant in 2009.

\section{REFERENCES}

Aaronson, S.A. and Todaro, G.J. (1968). Development of 3T3-like lines from Balb-c mouse embryo cultures: transformation susceptibility to SV40. J. Cell. Physiol., 72, 141-148.

Alépée, N., Tornier, C., Robert, C., Amsellem, C., Roux, M.H., Doucet, O., Pachot, J., Méloni, M. and de Brugerolle de Fraissinette, A. (2010). A catch-up validation study on reconstructed human epidermis (SkinEthic RHE) for full replacement of the Draize skin irritation test. Toxicol. In Vitro, 24, 257-266

Ball, P., Mandell, L. and Toillotson, G. (1999). Comparative tolerability of the newer fluoroquinolone antibacterials. Drug Saf., 21, 407-421.

Brantom, P.G., Bruner, L.H., Chamberlain, M., de Silva, O., Dupis, J., Earl, L.K., Lovell, D.P., Pape, W.J.W., Uttley, M., Bagley, D.M., et al. (1997). A summary of the COPILA international validation study on alternatives to the Draize Rabbit Eye irritation test. Toxicol. In Vitro, 11, 141-179.

Calvin, G. (1992). New approaches to the assessment of eye and skin irritation. Toxicol. Lett., 64, 157-164.

Chetlat, A.-A., Albertini, S. and Gocke, E. (1996). The photomutagenicity of fluoroquniolones in tests for gene mutation, chromosomal aberration test, gene conversion and DNA breakage (Comet assay). Mutagenesis, 11, 497-504.

Coquette, A., Berna, N., Vandenbosch, A., Rosdy, M., De Wever, B. and Poumay, Y. (2003). Analysis of interleukin-1a (IL-1a) and interleukin-8 (IL-8) expression and release in in vitro reconstructed human epidermis for the prediction of in vivo skin irritation and/or sensitization. Toxicol. In Vitro, 17, 311-321.

Corsini, E., Bruccoleri, A., Marinovich, M. and L., G.C. (1996). Corsini, E., A. Bruccoleri, M. Marinovich, and C.L. Galli. Endogenous interleukin-1 alpha associated with skin irritation induced by tributyltin. Toxicol. Appl. Pharmacol., 138, 268274.

Fitzhugh, O.G., Woodard, G. and Braun, H.A. (1946). The toxicities of compounds related to 2,3-dimercaptopropanol (BAL) with a note on their relative therapeutic efficiency. J. Pharmacol. Exp. Ther, 87, 23-27.

Freeberg, F.E., Nixon, G.A., Reer, P.J., Weaver, J.E., Bruce, R.D., Griffith, J.F. and Sanders, L.W.I. (1986). Human and rabbit eye responses to chemical insult. Fundam. Appl. Toxicol., 7, 626634.

Gautheron, P., Dukic, M., Alix, D. and Sina, J.F. (1992). Bovine corneal opacity and permeability test: An in vitro assay of ocular irritancy. Fundam. Appl. Toxicol., 18, 442-449.

Griffith, J.F., Nixon, G.A., Bruce, R.D., Reeer, P.J. and Bannan, E.A. (1980). Dose-response studies with chemical irritants in the albino rabbis eye as a basis for selecting optimum testing conditions for predicting hazard to the humane eye. Toxicol. Appl. Pharmacol., 55, 501-512.

Hatao, M., Murakami, N., Sakamoto, K., Ohnuma, M., Matsushige, C., Kakishima, H., Ogawa, T., Kolima, H., Matsukawa, K., Masuda, K., Chiba, K., Yoshizawa, K., Kaneko, T., Iwabuchi, Y., Matsushima, Y., Momma, J. and Ohno, Y. (1999). Interlaboratory validation of the in vitro eye irritation tests for cosmetic ingredients. (4) Haemoglobin denaturation test. Toxicol. In Vitro, 13, 125-137. 
Hong, M.-Y., Kim, J.-Y., Lee, Y.M. and Lee, M. (2005). Assessment of sensitivity of photo-chromsomal assay in the prediction of photo-carcinogenicity. J. Toxicol. Pub. Health, 21, 99105.

Jung, K.-M., Moon, J.-Y., Lee, S.-H., Kim, C.-W., Park, C.-B. and Kim, B.H. (2008). The validation of alternative methods of reconstructed human skin equivalents for the assessment of skin irritation. J. Altern. Ani. Exp., 2, 31-38.

Kim, J.-Y., Koh, W.S. and Lee, M. (2006). Validation of photocomet assay as a model for the prediction of photocarcinogenicity. J. Toxicol. Pub. Health, 22, 423-429.

Lee, H.-K., Noh, K.-J., Seok, S.-H., Baek, M.-W., Lee, H.-Y., Kim, D.-J., Na, Y.-R., Park, S.-H., Kim, B.H., Park, G.-R., Lee, J.-G. and Park, J.H. (2008). Establishment of the neutral red uptake assay as alternatives to the Draize test and its validation. J. Altern. Ani. Exp., 2, 5-9.

Luepke, N.P. (1985). Hen's egg chorioallantoic membrane test for irritation potential. Fd. Chem. Toxicol., 23, 287-291.

Mosman, T. (1983). Rapid colorimetric assay for cellular growth and survival: application to proliferation and cytotoxicity assays. J. Immunol. Methods, 65, 55-63.

NICEATM (2003). Test Method Protocol for BALB/c 3 T3 Neutral Red Uptake Cytotoxicity Assay Phase III - Validation Study (November 4, 2003).

OECD (2007). OECD Guidelines for the Testing of Chemicals (December, 2007) Draft proposal for a new guideline 'In Vitro Skin Irritation: Human Skin Model Test'.

Osborne, R., Perkins, M.A. and Roberts, D.A. (2000). In vitro method for eye and skin irritation testing. United States Patent 6020148 (http://www.freepatentsonline.com/6020148.html).
Pape, W.J., Pfannenbeckker, U. and Hoppe, U. (1987). Validation of the red blood cell test system as in vitro assay for the rapid screening of irritation potential of surfactants. Mol. Toxicol., 1, 525-536.

Prinsen, M.K. and Koeter, H.B. (1993). Justification of the enucleated eye test with eyes of slaughterhouse animals as an alternative to the Draize eye irritation test with rabbits. Fd. Chem. Toxicol., 31, 69-76.

Rosdy, M. and Clauss, L.C. (1990). Terminal epidermal differentiation of human keratinocytes grown in chemically defined medium on inert filter substrates at the air-liquid interface. $J$. Invest. Dermatol., 95, 409-414.

Spielmann, H., Liebsch, M., Moldenhauer, F., Holzhutter, H.-G. and de Silva, O. (1995). Modern biostatistical methods for assessing in vitro/in vivo correlation of severely eye irritating chemicals in a validation study of in vitro alternatives to the Draize eye test Toxicol. In Vitro, 9, 549-556.

Vinardell, M.P. and Mitjans, M. (2008). Alternative methods for eye and skin irritation tests: an overview. J. Pharm. Sci., 97, 4559.

Yang, X., Zhang, W., Yang, Y., Xiong, X., Xie, X. and Tan, X. (2007) Preliminary study on neutral uptake assay as an alternative method for eye irritation test. AATEX, 14, 509-514.

Yang, W. and Acosta, D. (1994). Cytotoxicity potential of surfactant mixtures evaluated by primary cultures of rabbit corneal epithelial cells. Toxicol. Lett., 70, 309-318.

York, M. and Steiling, W. (1998). A critical review of the assessment of eye irritation potential using the Draize rabbit eye test. J. Appl. Toxicol., 18, 233-240. 